\title{
Paratactical Tuning: An Agenda for the Use of Computers in Experimental Intonation
}

\author{
Larry Polansky \\ Computer Music Journal, Vol. 11, No. 1, Microtonality. (Spring, 1987), pp. 61-68.
}

Stable URL:

http://links.jstor.org/sici?sici=0148-9267\%28198721\%2911\%3A1\%3C61\%3APTAAFT\%3E2.0.CO\%3B2-4

Computer Music Journal is currently published by The MIT Press.

Your use of the JSTOR archive indicates your acceptance of JSTOR's Terms and Conditions of Use, available at http://www.jstor.org/about/terms.html. JSTOR's Terms and Conditions of Use provides, in part, that unless you have obtained prior permission, you may not download an entire issue of a journal or multiple copies of articles, and you may use content in the JSTOR archive only for your personal, non-commercial use.

Please contact the publisher regarding any further use of this work. Publisher contact information may be obtained at http://www.jstor.org/journals/mitpress.html.

Each copy of any part of a JSTOR transmission must contain the same copyright notice that appears on the screen or printed page of such transmission.

The JSTOR Archive is a trusted digital repository providing for long-term preservation and access to leading academic journals and scholarly literature from around the world. The Archive is supported by libraries, scholarly societies, publishers, and foundations. It is an initiative of JSTOR, a not-for-profit organization with a mission to help the scholarly community take advantage of advances in technology. For more information regarding JSTOR, please contact support@ jstor.org. 


\section{Larry Polansky}

Center for Contemporary Music

Music Department

Mills College

Oakland, California 94613 USA

\section{Paratactical Tuning: An Agenda for the Use of Computers in Experimental Intonation}

often clearly understood in the light of their broader intonational consequences (and possibilities), even by those who have developed them.

A second limiting factor is the set of two interrelated notions that (1) there is some set of "pure" or desirable intervals against which intonational schemas may be judged or "calibrated" and that (2) the choice of any given "scale," or fixed set of available pitches, necessitates intonational compromises and deviations from these desired tunings. For example, it is often assumed, when one describes the quality of a high-number equal temperament (like the popular twelve-tone, 31-tone, or 53-tone), that "goodness or badness" is based upon some "fit" to the ratios $5 / 4$ for its "major thirds," $3 / 2$ for its "perfect fifths," and so on.

It is less clear, of course, with these equal temperaments, what ratios are assumed as "ideal" for the "minor seventh" $(9 / 5,7 / 4, \ldots$ ? $)$, the "major second" $(10 / 9,9 / 8$, or even the $8 / 7$ which is so common in non-Western musics), and all of the other interval classes. Indeed, it is a significant assumption, in and of itself, that scales and tunings must approximate these particular interval classes. Why not, for example, have several "major thirds" (say the $81 / 64$, the $5 / 4$, and the $9 / 7$ ) to accommodate various musical situations?

Some composers and theorists who have experimented with equal temperaments have of course been cognizant of these limitations of fixed tunings. They have used this class of scales to form more sophisticated harmonic and tonal fabrics than simple approximations of a canonical Just intonation. It seems to me more commonly the case, however, that the use of the so-called microtonal divisions is motivated by either an interest in "modulation" or by mechanical and/or practical exigencies. In the case of modulation, it is important to note that equal-tempered modulation by definition will always lead to an equivalent set of intervals! In 
the case of practical matters, it is obvious that if one is refretting a guitar, or dividing a reference frequency, equal temperament is often easier.

Equal temperaments have proven to have some very exciting and expansive compositional uses, like the aforementioned equivalent modulation, and also in approximating complex harmonic spaces. James Tenney's recent work in 72-tone equal (see the section "Next" later), and Jim Horton's use of 171-tone equal (Horton 1986) are just two of many interesting examples. One of the primary advantages of equal temperaments is that they accommodate the "fixed-pitch" category of instruments. It is important to realize however, that all equal temperaments are also some subset of the class of fixed scales, mentioned previously, and thus create certain other musical problems.

Intonational systems that use a fixed set of pitches to try to "come close" to another ideal set could be referred to as static approximations. In general, static approximation tunings do not involve a great deal of ambiguity - there is usually one pitch that is closest to some other, not-present, desired pitch, and a syntactic relationship exists between that ideal and its representative in the actual sounding set. Another characteristic of (equal-tempered) static approximation tunings is the tendency to generate unwanted pitch material, since many intervals in the set are simply artifacts of the division process. A greater number of divisions of the octave is more likely to generate closer fits, but it also generates more unnecessary material-though often these other pitches can yield interesting music.

One of the oldest, and most overused examples of the need for the existence of these static approximations is the set of intonational dilemmas created in the simple diatonic Just scale. Here it is given in ratios to $1 / 1$ :

\section{$\begin{array}{llllllll}1 / 1 & 9 / 8 & 5 / 4 & 4 / 3 & 3 / 2 & 5 / 3 & 15 / 8 & 2 / 1\end{array}$}

This five-limit scale would seem to be one of the simplest ways to tune the "white keys" so as to maximize the acoustic consonance of the intervals - that is, use as many $5 / 4$ and $3 / 2$ ratios /and their inversions) as possible. However, a harmonic problem arises almost immediately in the perfect fifth built upon the supertonic. This creates the "wolf fifth" of 40/27, a quite-narrow interval lapproximately 680 cents) of the perfect fifth of $3 / 2$ (702 cents). In other words, a simple set of intonational assumptions, juxtaposed with a simple mechanical exigency (fixed-pitch keyboard), leads to a sort of contradiction. The contradiction depends upon the restrictive exigency, and when that no longer exists (as is the case today when the technology allows for time-variant keyboard tunings), we are less obligated to work in the tuning systems generated and evolved from that contradiction.

Where equal temperaments strive to approximate desired intervals, it is a simpler and more-elegant solution to allow for time- and context-variant scales. Precedents are clear, as this practice is quite ancient in instruments (and voices) of variable pitch. It could be argued that much of the history of Western intonational practice and evolution, including meantone temperament, well temperaments, and equal temperaments, is generated in part by this one basic problem-that in the key of $\mathrm{C}$, tuned Just, a perfect fifth on D precludes one on $G$, especially with the primacy of the twelve-note keyboard. In addition, any static approximation is likely to generate an interest in actually achieving the approximated intervals in some way. The motivations for approximation and compromise are obviated to a great extent, however, by recent technologies that give us the ability to change tunings accurately and quickly.

\section{Slendro and Paratact}

The next two sections of this article offer a few examples of methods used to circumvent the tuning dilemmas discussed in the first section. These methods predate the use of a computer to dynamically alter tuning systems "on the fly," and offer clear examples of what some of the goals are for tuning systems of all types. In addition, they suggest approaches that might be valuable for computer-based tuning systems.

The most familiar examples occur in the intonational history of Western art music. Meantone and well temperaments afford composers with sets of approximations. These provide useful tonal resources in a great number of tonal centers. More impor- 
tantly perhaps, these tunings fundamentally alter the sonic material depending on which tonal center(s) is (are) currently in use. Twelve-tone equal temperament can thus be described as a natural evolution of these earlier tunings, based on a higher priority for the criteria of equivalence of tonal centers over the importance of the approximation of canonical intervals.

Paratactical tuning systems are ones in which the intervals are highly context-dependent. Paratactical tunings are constructed to optimize multiple uses of a given interval. In some sense, most tunings would be considered paratactical, and I do not posit the term for exclusionary purposes, but rather with the intent of focusing attention on this particular criteria of scale formation. Paratactical tunings arise from the almost contrary criteria of (1) the practical need to use fixed tunings, and (2) the desire for complex and acoustically sound intonational systems.

These tuning systems seem to be a kind of sophisticated, almost poetic extension of fixed tuning systems like equal temperaments. In general, paratactical tunings have two important and related characteristics: (1) their intervallic relationships generate a great deal of ambiguity, and (2) it is often difficult to determine which actual pitch is approximating which ideal pitch. In a grammatical sense, their "syntax" is highly context-dependent (hence the term paratax, see the Appendix).

An interesting illustration can be found in even a cursory glance at central Javanese slendro tunings. Slendro is a five-note scale. Along with the seventone pelog, from which five-tone pathet (roughly, "modes") are derived, slendro is used widely throughout Indonesian music, but with no standardization of intonation. Tunings vary tremendously from musical culture to musical culture, and even from gamelan to gamelan in neighboring locales. Although there are several interesting criteria for the invention of a particular slendro /done usually when a gamelan is first constructed, although gamelan are often retuned), it is difficult to put these criteria into the language we usually use to describe tuning systems in the West.

One of the most interesting phenomena in some of the central Javanese slendro tunings is the man- ner in which "spiral tunings" are incorporated into the inner intervals. It is common in this tradition to tune octaves a bit wide, by as much as 36 cents. The stretched octave varies widely, and there is not much numerical information available pertaining to the extent and variation of this practice. However, a look at eight of the tunings from the classic Gadjah Mada study of Jogiakarta and Surakarta gamelan(Surjodiningrat, Sudariana, and Susanto 1972) shows that each octave is wide. The eight octaves are, in cents, from narrowest to widest: 1206, 1208, 1210, 1213, $1219,1224,1228$, and 1236. The average "stretch" is approximately 18 cents. This wide octave creates an unusual set of relationships possible for a given internal scale interval. For example, the fifth $(V)$ degree of the scale is some kind of "fifth" above the first (I), and some kind of fourth below the I' above it. In addition, it must be considered that the $2 / 1$ octave of low I has at least an implied psychoacoustic presence, and thus $\mathrm{V}$ has some kind of relationship to it, which is, admittedly, closely correlated to its relationship to $1 / 1$.

The next chart lists these three sets of relationships (in cents) for the eight tunings in the Gadjah Mada study. V is the fifth degree, I is the actual first degree, $2 * \mathrm{I}$ is the "true" octave not actually present, and I' is the actual high I.

$\begin{array}{ccc}\mathrm{V} / \mathrm{I} & 2 * \mathrm{I} / \mathrm{V} & \mathrm{I}^{\prime} / \mathrm{V} \\ 736 & 464 & 500 \\ 734 & 466 & 490 \\ 708 & 492 & 500 \\ 738 & 462 & 490 \\ 719 & 481 & 491 \\ 701 & 499 & 505 \\ 725 & 475 & 494 \\ 715 & 485 & 508\end{array}$

Some quite unusual relationships are thus formed, with a clear trend towards keeping the $\mathrm{I}^{\prime} / \mathrm{V}$ close to a Just fourth $(4 / 3=498$ cents), consequently, sacrificing the intonation of a $3 / 2$ fifth. The average value of the fifth is about 722 cents, suggesting to me, because of the preponderance of seven-limit ratios in the music (Polansky 1985), an approximation of the 32/21 (729 cents) to the low I as a kind of target. This implies that the $\mathrm{V}$ is a $21 / 16$ below the $2 / 1$. 
It is beyond the scope of this article to completely investigate these scales /see Polansky 1985 for a more complete examination), but if one considers these intervals in relation to the other intervals in the scale, which also have their own "bipolar" tendencies, it becomes apparent that some intervals relate more simply to the "phantom octave" than to $I^{\prime}$. This is supported by the fact that the intervals in the scales tend to center most often around the simple septimal ratios of $7 / 6,8 / 7,12 / 7$, and $147 /$ 128. For example, the average interval of VI/V $(243$ cents) in these slendro is within a few cents of a $147 / 128(21 / 16 * 7 / 4$, or a "perfect fourth above a minor seventh"). The average of VI is about 965 cents from low I, very close to the $7 / 4$ (969 cents).

The consistency of this intonational pattern suggests that the primary criteria for tuning $\mathrm{V}$ is to maintain a certain relationship with VI. This seems at least in part intuitive, because of the frequency of stepwise motion in central Javanese court music between those two degrees. For the tuning of six below I', the average value is about 254 cents /or about 946 cents from low I, as stated above), which suggests that VI is tuned not to $I^{\prime}$, but to $2 * \mathrm{I}(!)$, because of the stretched tuning ( 18 cents). The sixth degree begins to look very much like the $8 / 7$ below that "phantom" degree (which would be 1200 $[254+18]=964$, as compared to 969 for the true $8 / 7)$, an interval which would be in keeping with the rest of the intonational fabric.

This rather abstract exercise is less a study of Javanese tunings than an example of the way in which the tuning of an extremely limited set of fixed pitches (five!) can suggest, and in many cases actually be, a complex intonational schema, yielding a fertile harmonic and melodic environment. These tunings are paratactical in the truest sense, for how an interval sounds is inextricably related to its context-what precedes and succeeds it. In this sense it is also time dependent, or at least gives the illusion of being so. Some have suggested that these complex, implicational intonations are related, and even integral to, the important notion of "indirection" common in the art forms of central Java culture-the phenomenon of approaching a target by first moving away from it (Mitoma 1985). My own opinion is that this "indirection" also applies to certain pitches (like $2 *$ I) that are not even present in the tuning!

There are of course, non-fixed-pitch instruments in the gamelan (the rebab, the suling, the pesinden, and others) which often play in other scales entirely, simultaneously with the fixed-pitch instruments. In addition, the seven-tone pelog itself has complex modal characteristics, often in relationship to the slendro. An investigation of these aspects would be highly interesting, and I suspect, almost unbelievably complex. In other Southeast Asian musics, for example Khmer, many of these paratactical phenomena are also manifested in what are often assumed (I think mistakenly) to be equal heptatonic scales but which are most likely paratactical combinations of equal temperament, the "not quite seven-tone equal" $8 / 7$ (231 cents versus $240)$, and non-2/1 octaves.

\section{Paratact in "Northwest Asia"}

This section offers examples from the work of three contemporary composers who have utilized some of the flexibility of paratactical tuning systems. One of the most important points of view has been stated by Lou Harrison / the source of the quote in the title of this section), who long ago recognized the limitations of both fixed-pitch Just scales and static approximations. Harrison calls his approach free style, as opposed to strict style. In free style the successive iteration of desired ratios constructs the intonational fabric. This is exemplified in pieces like Arion's Leap, Simfony in Free Style, and At the Tomb of Charles Ives.

After only a brief study of intervals it becomes clear that there are two ways of composing with them: (1) arranging them into a fixed mode, or gamut, and then composing within that structure. This is Strict Style, and is the vastly predominant world method. However, another way is possible-(2) to freely assemble, or compose with whatever intervals one feels that he needs as he goes along. This is Free Style, and I used this method first in my Simfony in Free Style (Harrison, Music Primer, pp. 6-7). 
Fig. 1. Excerpt from Lou

Harrison's Simfony in Free

Style.

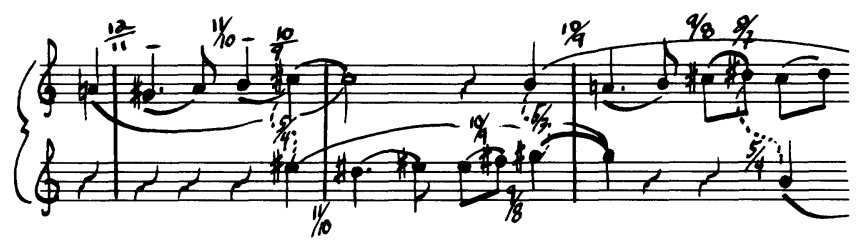

Figure 1 illustrates an example from Harrison's Simfony in Free Style, "a rising and 'expanding' sequence."

The difficulties of this method are formidableonly electronic instruments can implement it. Yet the intonational fabric achieved is far more complex than would be a "fixed gamut," since there are theoretically an infinite number of pitches derivable from a very limited set of ratios. It is in every sense a relational and paratactic tuning. It should be obvious that this method lends itself immediately to computer implementations where the tuning requirements are quite simple to realize-simpler, in some respects, than on acoustic instruments.

Another example exists in the very recent work of James Tenney, who uses a 72 -tone equal scale to approximate a seven-limit Just harmonic space, in pieces like Bridge (for two pianos in a microtonal tuning system), and Changes, 64 Studies for Six Harps.

The tuning system for Bridge is a slightly "tempered" or quasi-Just approximation of a "7limit" Just set of 22 pitches/octave, obtained by the use of incremental deviations of onesixth of a semitone from the standard 12-tone temperament (Tenney, instructions to Bridge).

Tenney represents this tuning system as a lattice in a three-dimensional ratio space (projected onto two dimensions), shown in Fig. 2, similar to the types of diagrams Ben Johnston also uses to represent his tuning systems.

Horizontal connecting lines represent tempered fifths or fourths $(3 / 2-2.0$ cents, $4 / 3$ +2.0 ), right-ascending lines quasi-Just major thirds or minor sixths $(5 / 4-3.0$ cents, $8 / 5$ +3.0 cents), and right-descending (solid) lines quasi-Just septimal minor sevenths or major seconds $(7 / 4-2.1$ cents, $8 / 7+2.1$ cents). Note that-because of the "temperament" - one pair of points in the lattice is identical to another pair (i.e., $8 / 5=45 / 28$ and $28 / 15=15 / 8$ ) (Tenney, instructions to Bridge).

Tenney is, strictly speaking, using a static approximation method here, but what makes it so unusual, and exemplary of the idea of a paratactical tuning, is that an equal-tempered scale is chosen specifically because many of its divisions have multiple, harmonically dependent functions. Note that Tenney, in giving cents deviations, is denoting the equidistance of the 72-tone approximations to two different (but very close) ratios.

A third example, perhaps the one most technologically relevant to the topic at hand, is the recent work of Harold Wagge in "intelligent keyboard" design (Wagge 1984, 1985). Wagge's seven-limit keyboard is an interesting step forward in keyboardtuning methodology, one that could easily be generalized to include more complex tunings. Wagge uses a logic circuit that adjusts the intonations of tertial relationships by 14 cents for the third, and by 31 cents for a septimal tuning when a "seventh" is sensed. One of the intents of this keyboard design is to allow the performer to simply play, while the keyboard performs the dynamic intonational adjustment that one would normally expect of a nonfixed-pitch ensemble of some sensitivity. Wagge's keyboard supports a limited number of intonation adjustments, namely the 5/4 third and the $7 / 4 \mathrm{sev}$ enth, but it can "handle" ninth chords. It seems to be designed so that any chord built upon tertiary intervals will be tuned in an expected fashion by his logic.

What is so striking to me about Wagge's idea is that it is the first example I know of an actual implementation of the solution to the problem elucidated in the first section of this article regarding any fixed Just tuning.

In a highly fluid tuning system such as this, what may be called the "supertonic dilemma" cannot occur. Thus, D1 [the note D adjusted by 14 cents-L.P.] is immediately available when the chord D1-F-Al [the latter the pitch A adjusted by the same 14 cents-L.P.] is played, but is as instantly changed to $\mathrm{D}$ when the chord $\mathrm{D}-\mathrm{G}-\mathrm{B} 1$ is played (Wagge 1985). 
Fig. 2. Tuning lattice from

James Tenney's Bridge. Reproduced courtesy of Sonic Art Editions.

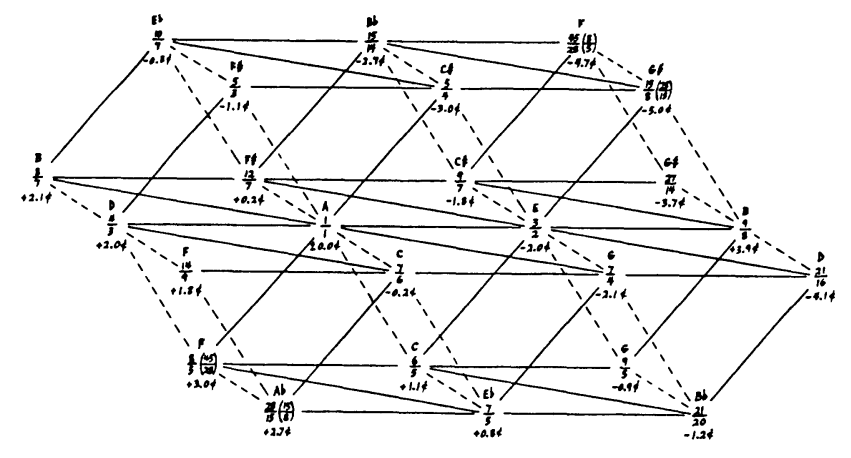

Next

This last section suggests an agenda for future experiment, taking into account what has been discussed so far. It is intended as an attempt to establish a "program" for the use of computer technology in intonational experimentation.

1. Elimination of Keyboard Primacy. It is clear that much of the history of intonation, and even current experimentation (like Wagge's) has been cognizant, if not downright obeisant, to the primacy of the twelve-note keyboard. Recent research and development in the area of input structures has been energetic in the computer music community. Some of the most successful techniques have included: mice, ultrasonic sensors, bio-sensors, movement sensors, and pitch, motion, position, timbre, and velocity/pressure to the Musical Instrument Digital Interface (MIDI) and/or voltage conversion on conventional and unconventional instruments. At the very simplest level, none of these input structures need invoke the "supertonic dilemma" /to use Wagge's terminology), nor any of its more complex manifestations.

There is now a liberating technology that need not contend with the contrary restrictions that have shaped much of the history of scales and tunings. What is needed instead is a mapping of controller to pitch space for each instance. Even more important is the development of a more general theory of how to achieve such mappings in powerful ways which are not controller-specific.

2. Dynamic Tunings. In an extension of my first point, it is no longer necessary to choose between the relative advantages of static approximations and paratactical tunings. Because of the ease and speed with which processors can retune (much faster than we can play, even at the relatively slow MIDI rates), we can employ the tuning we want, when we want it. There now exist several inexpensive, commercially available sound producing (and computing) machines that can be tuned (1) quickly and algorithmically in real time, (2) polyphonically, (3) in response to rapid stimuli, and (4) more or less as precisely as we wish. The Apple Macintosh and the Commodore Amiga (both of which have four, timbrally simple, tunable "voices") are two of the most widely used such machines at present. Now that in expensive, tunable MIDI synthesis devices (like the Casio CZ-101 and the Yamaha FB01) are being developed, composers and performers will begin to embrace and utilize these ideas more and more.

Static approximations and paratactical tunings still have specific and important uses and advantages. For example, static approximations are useful when conventional instruments are combined with dynamically tunable ones. In the case of paratactical tunings, it should not be overlooked that implication is often more interesting than statement. We probably would not want to design a gamelan such that the internal intervals change their tuning to achieve the "most important" ratio. These tunings are intimately involved with the form and content of their associated musics.

It is now practical, however, to use each tuning class-dynamic, static, or paratactic-when and if we want, for reasons more musically interesting, and more directly involved with compositional and musical intent than the primarily mechanical and practical reasons of the past. For example, the reasons for the genesis of twelve-tone equal temperament may no longer be relevant-making that system more useful for consideration as an experimental and/or historic tuning, like Just intonations.

3. Consonance and Dissonance. Theories of consonance and dissonance constitute perhaps the most essential field of research in the design of computerized tuning systems. In the most general sense, these theories should be able to specify computable distance functions in harmonic ratio spaces. Traditionally, consonance/dissonance evaluations 
have often been made according to some concept of "small-number ratios"-although the sizes and prime-number limits of these numbers have had a gradual historic evaluation. Psychoacoustically, there is much evidence to suggest that listener judgments of consonance and dissonance are closely related to the interactions of the partials of complex tones (Helmholtz 1954), as well as to the percentage of the critical bandwidth by which frequencies (fundamentals and partials) differ (Plomp and Levelt 1965; Dowling and Harwood 1986).

Composers like James Tenney (1986), Ben Johnston (1986), and others have been able to represent harmonic spaces so that distances can be computed along prime-number axes. One of the important ramifications of this concept is its stylistic independence. By specifying (1) the "priorities" (or perceptual weights) of various primes and (2) the desired distance along axes, a composer (or program) can construct any rational tuning system.

4. Distance Function in Harmonic Space. The need for a harmonic distance function becomes clear when we design machine decision-making processes for tuning systems. Wagge's logic circuit implements one such, and contains an implicit function which yields small distance values for $5 / 4$ thirds, 3/2 fifths, and larger values for septimal ratios. It returns very large distances for higher prime ratios $(11,13$, etc.). In fact, these values do not occur at all! Yet it is not difficult to see how a general algorithm might be constructed which would perform the following tasks:

1. Always keep track of the absolute ratio being sounded to some reference $(1 / 1)$

2. Move harmonically along prime axes according to user specification or system computation of prime priority (for example, the user might specify a tuning with only two axes, 7 and 13 !)

3. Move harmonically through the entire harmonic space according to user specification or system computation of absolute distance from current position

For tasks 2 and 3, the algorithm might resemble a simple "city-block" metric, where distances between ratios are the sum of the number of nodes that the system has to traverse to arrive at the target. For example, the distance between $5 / 4$ and $3 / 2$ could be computed like this:

$5 / 4$ is one node away from $1 / 1$ on the 5 axis and $3 / 2$ is one node away from $1 / 1$ on the 3 axis so $5 / 4$ is two (nodes) away from $3 / 2$

Some of the useful properties of this harmonic algorithm are (1) they are well defined, (2) they can be quickly and easily customized to reflect the system and/or composer's harmonic intents (either in a predetermined fashion or "on the fly"), and (3) the "city-block" distance function can easily incorporate weights for its axes to allow for prioritization of primes (these weights could of course be altered dynamically as well!).

Another method of achieving complex machine tunings is to use a large lookup table of scale values or frequencies; this is certainly the most common approach. In this algorithm, the ratios or frequencies are stored in some predetermined consonance or scalar order, and can be reordered dynamically by the user or by the system. This approach is simpler, and essentially implements the idea of fixed approximation. On the other hand, the use of a distance function on a continually computed tuning space, mentioned previously, roughly corresponds to a combination of Harrison's "free style," Tenney's and Johnston's " $n$-dimensional ratio lattice," and Wagge's "intelligent keyboard." This latter method is one which, in some sense, perceives harmonic distance, and represents an enormous and fertile set of experimental intonational possibilities on the computer.

\section{References}

Alter, R. 1981. The Art of Biblical Narrative. New York: Basic Books.

Dowling, W. J., and D. L. Harwood. 1986. Music Cognition. Orlando: Academic Press.

Harrison, L. Simfony in Free Style. New York: Editions Peters.

Harrison, L. "Precision Piece: A Phrase for Arion's Leap." Aptos. Hermes Beard Press. (Published in Ear $1: 1$, 1973.) 
Harrison, L. At the Tomb of Charles Ives. New York: Peer Publishing.

Harrison, L. 1971. Music Primer. New York: Edition Peters.

Helmholtz, H. 1954. On the Sensations of Tone. (Originally published 1877.) Trans. A. J. Ellis. New York: Dover.

Horton, J. 1986. "Horton Hears a Who (-le Number Ratio)." 1/1 2(2): 11-14.

Johnston, B. 1986. "Rational Structure in Music." 1/1 2(3): $12-15$.

Mitoma, J. 1985. "Aesthetic Form and Process in Central Java: Time, Space, and Force." Presented at the Second Annual All University of California Conference on Southeast Asia, University of California at Berkeley, April 12-15, 1985.

Plomp, R., and W. Levelt, 1965. "Tonal Consonance and Critical Bandwidth." Journal of the Acoustical Society of America 38:548-560.

Polansky, L. 1985. "Interval Sizes in Javanese Slendro." Balungan 1(2):9-11.

Surjodiningrat, W., P. Sudarjana, and A. Susanto. 1972. Tone Measurements of Outstanding Javanese Gamelans in Jogiakarta and Surakarta. Jogiakarta: Gadjah Mada University Press.

Tenney, J. 1984. Bridge for two pianos. Baltimore: Sonic Art Editions.

Tenney, J. 1986. Changes: 64 Studies for Six Harps. Baltimore: Sonic Art Editions.

Wagge, H. M. 1984. "The Varitonal Scale of Primetones, Pentones and Septones." EAR 9(1):2-9.

Wagge, H. M. 1985. "The Intelligent Keyboard." $1 / 1$ 1(4): 1, $12-13$.

\section{Appendix: Paratactic and Syntactic}

I am extending somewhat the precise, dictionary meaning of the words paratactic and syntactic |"The coordination of grammatical elements such as phrases or clauses, without the use of coordinating elements such as conjunctions," and "The way in which words are put together to form phrases and sentences," respectively, from the American Heritage Dictionary of the English Language, 1969). My use is in part inspired by the literary connotations of these terms, especially the thinking of Robert Alter, whose narrative analyses of the Old Testament give important insights into the artistic applications of these grammatical ideas.

Because it is a literature that breaks away from the old cosmic hierarchies, the Bible switches from a reliance on metaphor to a reliance on metonymy. Schneidau attempts to summarize this whole contrast in an aphorism: "Where myth is hypotactic metaphors, the Bible is paratactic metonymies." That is, where myth involves a set of equivalencies arranged in some system of subordination, the Bible offers series of contiguous terms arranged in sequence without a clear definition of the link between one term and the next (Alter 1981, p. 26).

In this light I invoke the word paratact to describe a situation in which cause and effect are not unambiguously specified, but which are sometimes clarified by a larger context. A simple textual example might be the difference between "I feel so bad 'cause my baby left me this morning" (syntact) and "I feel so bad, my baby left me this morning" (paratact). 